\title{
WEAK-STAR PROPERTIES OF HOMOMORPHISMS FROM WEIGHTED CONVOLUTION ALGEBRAS ON THE HALF-LINE
}

\author{
THOMAS VILS PEDERSEN
}

\author{
(Received 22 January 2009; accepted 21 April 2010) \\ Communicated by G. A. Willis
}

\begin{abstract}
Let $L^{1}(\omega)$ be the weighted convolution algebra $L_{\omega}^{1}\left(\mathbb{R}^{+}\right)$on $\mathbb{R}^{+}$with weight $\omega$. Grabiner recently proved that, for a nonzero, continuous homomorphism $\Phi: L^{1}\left(\omega_{1}\right) \rightarrow L^{1}\left(\omega_{2}\right)$, the unique continuous extension $\widetilde{\Phi}: M\left(\omega_{1}\right) \rightarrow M\left(\omega_{2}\right)$ to a homomorphism between the corresponding weighted measure algebras on $\mathbb{R}^{+}$ is also continuous with respect to the weak-star topologies on these algebras. In this paper we investigate whether similar results hold for homomorphisms from $L^{1}(\omega)$ into other commutative Banach algebras. In particular, we prove that for the disc algebra $A(\overline{\mathbb{D}})$ every nonzero homomorphism $\Phi: L^{1}(\omega) \rightarrow A(\overline{\mathbb{D}})$ extends uniquely to a continuous homomorphism $\widetilde{\Phi}: M(\omega) \rightarrow H^{\infty}(\mathbb{D})$ which is also continuous with respect to the weak-star topologies. Similarly, for a large class of Beurling algebras $A_{v}^{+}$on $\overline{\mathbb{D}}$ (including the algebra of absolutely convergent Taylor series on $\overline{\mathbb{D}}$ ) we prove that every nonzero homomorphism $\Phi: L^{1}(\omega) \rightarrow A_{v}^{+}$extends uniquely to a continuous homomorphism $\widetilde{\Phi}: M(\omega) \rightarrow A_{v}^{+}$which is also continuous with respect to the weak-star topologies.
\end{abstract}

2000 Mathematics subject classification: primary 46J10; secondary 30H05, 46E30, 47B38.

Keywords and phrases: homomorphisms, weighted convolution algebras, weak-star continuity, function algebras.

\section{Introduction}

Define $\mathbb{R}^{+}=[0, \infty)$. We let $L^{1}\left(\mathbb{R}^{+}\right)$denote the Banach space of (equivalence classes of) integrable functions $h$ on $\mathbb{R}^{+}$with the norm

$$
\|h\|=\int_{0}^{\infty}|h(t)| d t .
$$

In this paper we will work with weighted analogues of $L^{1}\left(\mathbb{R}^{+}\right)$. We refer to Grabiner [4, 5] for further details about the definitions and results summarized below.

A positive Borel function $\omega$ on $\mathbb{R}^{+}$is called an algebra weight if:

(a) $\quad \omega$ and $1 / \omega$ are locally bounded on $\mathbb{R}^{+}$;

(b) $\omega$ is right continuous on $\mathbb{R}^{+}$;

(C) 2010 Australian Mathematical Publishing Association Inc. 1446-7887/2010 \$16.00 
(c) $\omega$ is submultiplicative, that is, $\omega(t+s) \leq \omega(t) \omega(s)$ for all $t, s \in \mathbb{R}^{+}$;

(d) $\omega(0)=1$.

We then define $L^{1}(\omega)$ as the weighted space of all the functions $h$ on $\mathbb{R}^{+}$for which $h \omega \in L^{1}\left(\mathbb{R}^{+}\right)$with the inherited norm

$$
\|h\|_{\omega}=\int_{0}^{\infty}|h(t)| \omega(t) d t .
$$

(For convenience we will henceforth omit all subscripts on norms in this paper.) It is well known that, equipped with the usual convolution product

$$
(h * k)(t)=\int_{0}^{t} h(s) k(t-s) d s
$$

for all $t \in \mathbb{R}^{+}$and $h, k \in L^{1}(\omega)$, the space $L^{1}(\omega)$ is a commutative Banach algebra.

Similarly, the space $M(\omega)$ of locally finite complex Borel measures $\mu$ on $\mathbb{R}^{+}$for which

$$
\|\mu\|=\int_{\mathbb{R}^{+}} \omega(t) d|\mu| t<\infty
$$

is a Banach algebra under convolution and contains $L^{1}(\omega)$ as a closed ideal. (We integrate over $\mathbb{R}^{+}$, that is, $[0, \infty)$, since $\mu$ may have nonzero mass at 0 .) Hence every measure $\mu \in M(\omega)$ defines a multiplier $T_{\mu}$ on $L^{1}(\omega)$ by $T_{\mu} h=\mu * h$ for all $h \in L^{1}(\omega)$ and this identifies $M(\omega)$ isometrically isomorphic with the multiplier algebra $\operatorname{Mul}\left(L^{1}(\omega)\right)$ of $L^{1}(\omega)$. This induces a strong topology on $M(\omega)$ in which a net $\left(\mu_{\alpha}\right)$ tends to 0 if and only if $\mu_{\alpha} * h \rightarrow 0$ for every $h \in L^{1}(\omega)$. Moreover, if we denote by $C_{0}(1 / \omega)$ the Banach space of all continuous functions $g$ on $\mathbb{R}^{+}$for which $g / \omega$ is bounded and vanishes at infinity, with the norm

$$
\|g\|=\sup _{t \in \mathbb{R}^{+}} \frac{|g(t)|}{\omega(t)},
$$

then $M(\omega)$ is isometrically isomorphic to the dual space of $C_{0}(1 / \omega)$, and the duality is defined by

$$
\langle g, \mu\rangle=\int_{\mathbb{R}^{+}} g(t) d \mu(t)
$$

for all $g \in C_{0}(1 / \omega)$ and $\mu \in M(\omega)$. We thus have three different topologies on $M(\omega)$, namely the norm, strong and weak-star topologies. In the unweighted case where $\omega \equiv 1$, we use the notation $M\left(\mathbb{R}^{+}\right)$and $C_{0}\left(\mathbb{R}^{+}\right)$for $M(\omega)$ and $C_{0}(1 / \omega)$, respectively.

In order to present the inspiration for this paper we recall a result of Grabiner. For all $t \in \mathbb{R}^{+}$, we denote by $\delta_{t}$ the unit point measure at $t$. Then $\left(\delta_{t}\right)_{t \in \mathbb{R}^{+}}$is a strongly continuous semigroup in $M(\omega)$. Also, we refer to [6] for the general theory of Bochner integrals.

TheOREM 1.1 [4, Theorems 3.4 and 3.6]. Let $\omega_{1}$ and $\omega_{2}$ be algebra weights and let $\Phi: L^{1}\left(\omega_{1}\right) \rightarrow L^{1}\left(\omega_{2}\right)$ be a nonzero continuous homomorphism. Then $\Phi$ has a unique extension to a continuous homomorphism $\widetilde{\Phi}: M\left(\omega_{1}\right) \rightarrow M\left(\omega_{2}\right)$. Moreover, if we define $v^{t}=\widetilde{\Phi}\left(\delta_{t}\right)$ for all $t \in \mathbb{R}^{+}$, then $\left(v^{t}\right)_{t \in \mathbb{R}^{+}}$is a semigroup in $M\left(\omega_{2}\right)$ which is 
strongly continuous in $(0, \infty)$ and

$$
\widetilde{\Phi}(\mu)=\int_{\mathbb{R}^{+}} v^{t} d \mu(t)
$$

for all $\mu \in M\left(\omega_{1}\right)$, where the integrals exist as strong Bochner integrals in $M\left(\omega_{2}\right)$, that is,

$$
\widetilde{\Phi}(\mu) * h=\int_{\mathbb{R}^{+}} v^{t} * h d \mu(t)
$$

exists as a Bochner integral for all $\mu \in M\left(\omega_{1}\right)$ and $h \in L^{1}\left(\omega_{2}\right)$.

This result is closely related to the notion of a standard homomorphism [3]. A homomorphism $\Phi: L^{1}\left(\omega_{1}\right) \rightarrow L^{1}\left(\omega_{2}\right)$ is called standard if, whenever $h \in L^{1}\left(\omega_{1}\right)$ and $L^{1}\left(\omega_{1}\right) * h$ is dense in $L^{1}\left(\omega_{1}\right)$, then $L^{1}\left(\omega_{2}\right) * \Phi(h)$ is dense in $L^{1}\left(\omega_{2}\right)$. In [3, Theorem 2.2], it is proved that a nonzero homomorphism $\Phi$ is standard if and only if $\left(\nu^{t}\right)_{t \in \mathbb{R}^{+}}$is strongly continuous at 0 , which in turn holds if and only if $\widetilde{\Phi}$ is strongly continuous (with $v^{t}$ and $\widetilde{\Phi}$ as in Theorem 1.1). It is still an open question whether every nonzero homomorphism $\Phi: L^{1}\left(\omega_{1}\right) \rightarrow L^{1}\left(\omega_{2}\right)$ is standard, that is, whether the extension $\widetilde{\Phi}$ is automatically strongly continuous. However, Grabiner recently proved that $\widetilde{\Phi}$ is automatically weak-star continuous.

THEOREM 1.2 [5, Theorem 1.1]. Let $\Phi: L^{1}\left(\omega_{1}\right) \rightarrow L^{1}\left(\omega_{2}\right)$ be a nonzero continuous homomorphism, where $\omega_{1}$ and $\omega_{2}$ are algebra weights, and let $\widetilde{\Phi}: M\left(\omega_{1}\right) \rightarrow M\left(\omega_{2}\right)$ be the unique extension given by Theorem 1.1. Then $\widetilde{\Phi}$ is weak-star continuous.

\section{Extending homomorphisms into general algebras}

The aim of this paper is to consider possible generalizations of Theorem 1.2 for homomorphisms from $L^{1}(\omega)$ into other Banach algebras.

In [8, Proposition 1.1], we proved the following result, with $L^{1}\left(\mathbb{R}^{+}\right)$instead of $L^{1}(\omega)$, which shows that a homomorphism from $L^{1}(\omega)$ into an arbitrary commutative Banach algebra $\mathcal{B}$ can be extended to a homomorphism from $M(\omega)$ into a suitable extension of $\mathcal{B}$. For a closed ideal $\mathcal{C}$ in $\mathcal{B}$ we let $\operatorname{Mul}(\mathcal{C})$ be the multiplier algebra of $\mathcal{C}$ consisting of those bounded linear operators $T$ on $\mathcal{C}$ for which $T(a b)=a T(b)$ for all $a, b \in \mathcal{C}$.

PROPOSITION 2.1. Let $\mathcal{B}$ be a commutative Banach algebra, let $\omega$ be an algebra weight and let $\Phi: L^{1}(\omega) \rightarrow \mathcal{B}$ be a nonzero, continuous homomorphism. Then the subspace $\mathcal{C}=\Phi\left(L^{1}(\omega)\right) \mathcal{B}$ is a closed ideal in $\mathcal{B}$ and $\Phi$ extends to a homomorphism $\widetilde{\Phi}: M(\omega) \rightarrow \operatorname{Mul}(\mathcal{C})$ which is continuous with respect to both the norm and strong topologies. If $\mathcal{B}$ is an integral domain, then the extension of $\Phi$ is unique. Furthermore, if $v^{t}=\widetilde{\Phi}\left(\delta_{t}\right)$, then $\left(v^{t}\right)_{t \in \mathbb{R}^{+}}$is a strongly continuous semigroup in $\operatorname{Mul}(\mathcal{C})$, and $\left\|v^{t}\right\| \leq\|\widetilde{\Phi}\| \cdot \omega(t)$ for all $t \in \mathbb{R}^{+}$and $\widetilde{\Phi}(\mu)$ is given by the strong Bochner integral

$$
\widetilde{\Phi}(\mu)=\int_{\mathbb{R}^{+}} v^{t} d \mu(t)
$$


for all $\mu \in M(\omega)$, that is,

$$
\widetilde{\Phi}(\mu) c=\int_{\mathbb{R}^{+}} v^{t} c d \mu(t)
$$

exists as a Bochner integral for all $\mu \in M(\omega)$ and $c \in \mathcal{C}$.

The proof of Proposition 2.1 is basically the same as that of [8, Proposition 1.1]. The main ideas are as follows. The algebra $L^{1}(\omega)$ has a bounded approximate identity [4, Theorem 2.2], so Cohen's factorization theorem (see, for instance, [1, Corollary 2.9.26]) shows first that $\mathcal{C}$ is a closed ideal in $\mathcal{B}$, and second that for all $c \in \mathcal{C}$ there exist $h \in L^{1}(\omega)$ and $b \in \mathcal{C}$ such that $c=\Phi(h) b$. We then use an idea of Esterle [2, Proposition 5.2], and define

$$
\widetilde{\Phi}(\mu) c=\Phi(\mu * h) b
$$

for all $\mu \in M(\omega)$ and check that $\widetilde{\Phi}$ has the required properties. The uniqueness of the extension, when $\mathcal{B}$ is an integral domain, follows as in the proof of [4, Theorem 3.6].

For a nonzero, continuous homomorphism $\Phi: L^{1}\left(\omega_{1}\right) \rightarrow L^{1}\left(\omega_{2}\right)$ it follows from [3, Theorem 2.2] that $\Phi$ is standard if and only if the ideal $\mathcal{C}=\Phi\left(L^{1}\left(\omega_{1}\right)\right) L^{1}\left(\omega_{2}\right)$ is equal to $L^{1}\left(\omega_{2}\right)$. For standard homomorphisms, Grabiner's extension [4, Theorem 3.4] thus follows from Proposition 2.1.

In the following sections, we consider some concrete examples of the commutative Banach algebra $\mathcal{B}$. In these cases, the multiplier algebra $\operatorname{Mul}(\mathcal{C})$ sits naturally as a closed subspace of a certain dual space $Y^{*}$, and we show that the extension $\widetilde{\Phi}$ from Proposition 2.1 is weak-star continuous regarded as a map from $M(\omega)$ to $Y^{*}$. This is done by verifying, in these particular cases, the condition in the following more general result which allows us to exchange the order of duality and integration.

PROPOSITION 2.2. Let $\mathcal{B}$ be a commutative Banach algebra and let $\omega$ be an algebra weight. Moreover, let $\Phi: L^{1}(\omega) \rightarrow \mathcal{B}$ be a nonzero, continuous homomorphism and let

$$
\widetilde{\Phi}(\mu)=\int_{\mathbb{R}^{+}} v^{t} d \mu(t)
$$

for all $\mu \in M(\omega)$ be the extension to a homomorphism from $M(\omega)$ to $\operatorname{Mul}(\mathcal{C})$ given by Proposition 2.1. Suppose that $\operatorname{Mul}(\mathcal{C})$ is a closed subspace of a dual space $Y^{*}$, where $Y$ is a Banach space, and that the integrals

$$
\widetilde{\Phi}(\mu)=\int_{\mathbb{R}^{+}} v^{t} d \mu(t)
$$

for all $\mu \in M(\omega)$ exist as weak-star integrals, that is,

$$
\int_{\mathbb{R}^{+}}\left\langle y, v^{t}\right\rangle d \mu(t)
$$

exists and is equal to $\langle y, \widetilde{\Phi}(\mu)\rangle$ for all $\mu \in M(\omega)$ and $y \in Y$. Then the following conditions are equivalent. 
(a) The homomorphism $\widetilde{\Phi}: M(\omega) \rightarrow Y^{*}$ is weak-star continuous.

(b) The semigroup $\left(v^{t}\right)_{t \in \mathbb{R}^{+}}$is weak-star continuous in $Y^{*}$ and $v^{t} / \omega(t) \rightarrow 0$ weakstar in $Y^{*}$ as $t \rightarrow \infty$.

(c) For all $y \in Y$ and $t \in \mathbb{R}^{+}$, let

$$
(T y)(t)=\left\langle y, v^{t}\right\rangle
$$

Then $T y \in C_{0}(1 / \omega)$ for all $y \in Y$, and the map $T: Y \rightarrow C_{0}(1 / \omega)$ is a bounded linear operator and $T^{*}=\widetilde{\Phi}$.

ProOF. We show that (a) implies (b), that (b) implies (c), and that (c) implies (a).

Assume that (a) holds. Now $\left(\delta_{t}\right)_{t \in \mathbb{R}^{+}}$is weak-star continuous in $M(\omega)$, so $\left(v^{t}\right)_{t \in \mathbb{R}^{+}}$ is weak-star continuous in $Y^{*}$. Also,

$$
\left\langle g, \delta_{t} / \omega(t)\right\rangle=g(t) / \omega(t) \rightarrow 0 \quad \text { as } t \rightarrow \infty
$$

for $g \in C_{0}(1 / \omega)$, and so $\delta_{t} / \omega(t) \rightarrow 0$ weak-star in $M(\omega)$ as $t \rightarrow \infty$. Hence

$$
v^{t} / \omega(t)=\widetilde{\Phi}\left(\delta_{t} / \omega(t)\right) \rightarrow 0
$$

weak-star in $Y^{*}$ as $t \rightarrow \infty$.

Next, assume that (b) holds. For all $y \in Y$, clearly $T y \in C_{0}(1 / \omega)$ and

$$
\|T y\|=\sup _{t \in \mathbb{R}^{+}}\left|\left\langle y, \frac{v^{t}}{\omega(t)}\right\rangle\right| \leq \sup _{t \in \mathbb{R}^{+}} \frac{\left\|v^{t}\right\|}{\omega(t)}\|y\|,
$$

so $T: Y \rightarrow C_{0}(1 / \omega)$ is a bounded linear operator. For all $\mu \in M(\omega)$ and $y \in Y$, it follows from the assumption that

$$
\langle y, \widetilde{\Phi}(\mu)\rangle=\left\langle y, \int_{\mathbb{R}^{+}} v^{t} d \mu(t)\right\rangle=\int_{\mathbb{R}^{+}}\left\langle y, v^{t}\right\rangle d \mu(t)=\langle T y, \mu\rangle,
$$

so $T^{*}=\widetilde{\Phi}$.

Finally, assume (c) holds. Since $\widetilde{\Phi}=T^{*}$ with $T$ continuous, it follows that $\widetilde{\Phi}$ is weak-star continuous.

The examples of commutative Banach algebras $\mathcal{B}$ in the following sections are all semisimple. Consequently there only exist nonzero homomorphisms $\Phi: L^{1}(\omega) \rightarrow \mathcal{B}$ if $L^{1}(\omega)$ is semisimple, which is equivalent to the condition $\lim _{t \rightarrow \infty} \omega(t)^{1 / t}>0$ (see [1, Theorem 4.7.27]). We will therefore assume this to be the case in the rest of the paper.

\section{Homomorphisms into the disc algebra}

Let $A(\overline{\mathbb{D}})$ be the disc algebra, which consists of functions analytic on the open unit disc $\mathbb{D}$ and continuous on $\overline{\mathbb{D}}$. For a homomorphism $\Phi: L^{1}(\omega) \rightarrow A(\overline{\mathbb{D}})$ we can 
use the ideal structure in $A(\overline{\mathbb{D}})$ to get a more precise version of Proposition 2.1. Let $\mathcal{C}=\Phi\left(L^{1}(\omega)\right) A(\overline{\mathbb{D}})$ and let $E$ be the hull of $\mathcal{C}$, that is,

$$
E=\{z \in \overline{\mathbb{D}}: g(z)=0 \text { for every } g \in \mathcal{C}\} .
$$

Clearly,

$$
E=\left\{z \in \overline{\mathbb{D}}: \Phi(h)(z)=0 \text { for every } h \in L^{1}(\omega)\right\} .
$$

Since $\mathcal{C}$ contains a nonzero semigroup, the greatest common divisor of the inner factors in $\mathcal{C}$ is zero, so $E$ is contained in the unit circle $\mathbb{T}$ and

$$
\mathcal{C}=I(E)=\{g \in A(\overline{\mathbb{D}}): g=0 \text { on } E\}
$$

by the Rudin-Beurling theorem (see, for instance, [7, p. 85]).

Let $H^{\infty}(\mathbb{D})$ be the Banach algebra of bounded analytic functions on $\mathbb{D}$ and, for a closed set $E \subseteq \mathbb{T}$, let

$$
A(\overline{\mathbb{D}} \backslash E)=\left\{f \in H^{\infty}(\mathbb{D}): f \text { extends continuously to } \overline{\mathbb{D}} \backslash E\right\} .
$$

Then $A(\overline{\mathbb{D}} \backslash E)$ is a closed subalgebra of $H^{\infty}(\mathbb{D})$. Moreover, it is easily seen that

$$
\iota(f) g=f g,
$$

for all $f \in A(\overline{\mathbb{D}} \backslash E)$ and $g \in I(E)$, is an isometric isomorphism between $A(\overline{\mathbb{D}} \backslash E)$ and $\operatorname{Mul}(I(E))$ which induces a strong topology on the algebra $A(\overline{\mathbb{D}} \backslash E)$.

If we combine these observations with Proposition 2.1 we obtain most of Theorem 3.1 below. However, the theorem can also be obtained by using the more direct approach of [8, pp. 530-533], since the argument given in [8] for $L^{1}\left(\mathbb{R}^{+}\right)$also holds in the weighted case.

THEOREM 3.1. Let $\omega$ be an algebra weight, let $\Phi: L^{1}(\omega) \rightarrow A(\overline{\mathbb{D}})$ be a nonzero homomorphism and let

$$
E=\left\{z \in \overline{\mathbb{D}}: \Phi(h)(z)=0 \text { for every } h \in L^{1}(\omega)\right\} .
$$

Then the closed ideal $\mathcal{C}=\Phi\left(L^{1}(\omega)\right) A(\overline{\mathbb{D}})$ is equal to $I(E)$ and there exists a strongly continuous semigroup $\left(v^{t}\right)_{t \in \mathbb{R}^{+}}$in $A(\overline{\mathbb{D}} \backslash E)$ such that

$$
\widetilde{\Phi}(\mu)=\int_{\mathbb{R}^{+}} v^{t} d \mu(t)
$$

exists as a strong Bochner integral for all $\mu \in M(\omega)$, that is,

$$
\widetilde{\Phi}(\mu) g=\int_{\mathbb{R}^{+}} v^{t} g d \mu(t)
$$

exists as a Bochner integral for all $\mu \in M(\omega)$ and $g \in I(E)$. This defines a homomorphism $\widetilde{\Phi}: M(\omega) \rightarrow A(\overline{\mathbb{D}} \backslash E)$ that uniquely extends $\Phi$ and which is norm as well 
as strongly continuous. Moreover, $v^{t}(z)=e^{-t G(z)}$ for all $z \in \overline{\mathbb{D}} \backslash E$, where $G$ is analytic on $\mathbb{D}$ and continuous on $\overline{\mathbb{D}} \backslash E$, and

$$
\widetilde{\Phi}(\mu)(z)=\int_{\mathbb{R}^{+}} v^{t}(z) d \mu(t)
$$

for all $\mu \in M(\omega)$ and $z \in \overline{\mathbb{D}} \backslash E$.

One of the simplest examples of a homomorphism from $L^{1}\left(\mathbb{R}^{+}\right)$to $A(\overline{\mathbb{D}})$ can be constructed by means of the usual Laplace transform

$$
\mathcal{L}(h)(w)=\int_{0}^{\infty} h(t) e^{-w t} d t
$$

for all $h \in L^{1}\left(\mathbb{R}^{+}\right)$and $w \in \mathbb{C}$ with $\operatorname{Re} w \geq 0$ by letting

$$
\Lambda(h)(z)=\mathcal{L}(h)\left(\frac{1+z}{1-z}\right)=\int_{0}^{\infty} h(t) \exp \left(-t \frac{1+z}{1-z}\right) d t
$$

for all $h \in L^{1}\left(\mathbb{R}^{+}\right)$and $z \in \overline{\mathbb{D}} \backslash\{1\}$ (and $\Lambda(h)(1)=0$ ). In this way we obtain a homomorphism $\Lambda: L^{1}\left(\mathbb{R}^{+}\right) \rightarrow A(\overline{\mathbb{D}})$ with $E=\{1\}$ and for which the semigroup (of singular inner functions)

$$
v^{t}(z)=\exp \left(-t \frac{1+z}{1-z}\right)
$$

does not belong to $A(\overline{\mathbb{D}})$.

We mention in passing that the zero homomorphism does not have a unique extension. Denote the discrete part of a measure $\mu$ by $\mu_{d}$, and define

$$
\widetilde{\Phi}(\mu)(z)=\Lambda\left(\mu_{d}\right)(z)=\int_{\mathbb{R}^{+}} \exp \left(-t \frac{1+z}{1-z}\right) d \mu_{d}(t) ;
$$

then it can easily be seen that $\widetilde{\Phi}$ is a nonzero continuous homomorphism from $M\left(\mathbb{R}^{+}\right)$ to $A(\overline{\mathbb{D}})$ which is the zero homomorphism on $L^{1}\left(\mathbb{R}^{+}\right)$.

For a nonzero homomorphism $\Phi: L^{1}(\omega) \rightarrow A(\overline{\mathbb{D}})$, Theorem 3.1 provides a unique extension $\widetilde{\Phi}: M(\omega) \rightarrow A(\overline{\mathbb{D}} \backslash E)$. It is well known that $H^{\infty}(\mathbb{D})$ can be identified as the dual space of the quotient space $L^{1}(\mathbb{T}) / H_{0}^{1}(\mathbb{D})$, with the duality defined by

$$
\left\langle q+H_{0}^{1}(\mathbb{D}), f\right\rangle=\frac{1}{2 \pi} \int_{0}^{2 \pi} q\left(e^{i \theta}\right) f\left(e^{i \theta}\right) d \theta
$$

for all $q \in L^{1}(\mathbb{T})$ and $f \in H^{\infty}(\mathbb{D})$. For convenience we will henceforth simply write $q$ in place of $q+H_{0}^{1}(\mathbb{D})$.

Considering $A(\overline{\mathbb{D}} \backslash E)$ as a closed subspace of $H^{\infty}(\mathbb{D})$, we can thus regard $\widetilde{\Phi}$ as a map into the dual space $H^{\infty}(\mathbb{D})$ (which, furthermore, has the advantage compared to $A(\overline{\mathbb{D}} \backslash E)$ of being independent of $E$ and thus of $\Phi)$. We now aim to prove that $\widetilde{\Phi}$ is weak-star continuous. For homomorphisms from $L^{1}\left(\mathbb{R}^{+}\right)$this will be done in Theorem 3.6 and we will then use the following result to lift the result to the weighted case. 
PROPOSITION 3.2. Let $\omega$ be an algebra weight with the property that

$$
\lim _{t \rightarrow \infty} \omega(t)^{1 / t}=1,
$$

let $\Phi: L^{1}(\omega) \rightarrow A(\overline{\mathbb{D}})$ be a nonzero homomorphism, and let

$$
\widetilde{\Phi}(\mu)=\int_{\mathbb{R}^{+}} v^{t} d \mu(t)
$$

for all $\mu \in M(\omega)$, be the unique extension to a homomorphism from $M(\omega)$ to $H^{\infty}(\mathbb{D})$ given by Theorem 3.1. Then $\left(v^{t}\right)_{t \in \mathbb{R}^{+}}$is a bounded and strongly continuous semigroup in $A(\overline{\mathbb{D}} \backslash E)$, and if

$$
\breve{\Phi}(\mu)=\int_{\mathbb{R}^{+}} v^{t} d \mu(t)
$$

for all $\mu \in M\left(\mathbb{R}^{+}\right)$, then $\breve{\Phi}$ is an extension of $\Phi$ to a homomorphism from $M\left(\mathbb{R}^{+}\right)$ to $A(\overline{\mathbb{D}} \backslash E)$ which is norm as well as strongly continuous. Moreover, $\breve{\Phi}$ maps $L^{1}\left(\mathbb{R}^{+}\right)$ into $A(\overline{\mathbb{D}})$.

PROOF. Let $t \in \mathbb{R}^{+}$. Since $H^{\infty}(\mathbb{D})$ is a uniform algebra, $\left\|v^{t}\right\|=\|v\|^{t}$ (where $v=v^{1}$ ). Also, $\left\|v^{t}\right\| \leq\|\widetilde{\Phi}\| \omega(t)$, so $\|v\| \leq\|\widetilde{\Phi}\|^{1 / t} \omega(t)^{1 / t}$. Hence $\|v\| \leq 1$, so it follows that $\left(v^{t}\right)_{t \in \mathbb{R}^{+}}$is a bounded semigroup in $H^{\infty}(\mathbb{D})$. Since $\left(v^{t}\right)_{t \in \mathbb{R}^{+}}$is strongly continuous in $A(\overline{\mathbb{D}} \backslash E)$ by Theorem 3.1, it follows that

$$
\breve{\Phi}(\mu)=\int_{\mathbb{R}^{+}} v^{t} d \mu(t)
$$

exists as a strong Bochner integral for all $\mu \in M\left(\mathbb{R}^{+}\right)$and defines a continuous homomorphism $\breve{\Phi}: M\left(\mathbb{R}^{+}\right) \rightarrow H^{\infty}(\mathbb{D})$.

In order to prove that $\breve{\Phi}: M\left(\mathbb{R}^{+}\right) \rightarrow A(\overline{\mathbb{D}} \backslash E)$ is strongly continuous, we let $\left(\mu_{\alpha}\right)$ be a net in $M\left(\mathbb{R}^{+}\right)$which converges strongly to 0 , and let $g \in I(E)$. Since $I(E)$ has a bounded approximate identity, it follows from Cohen's factorization theorem that there exist $g_{1}, g_{2} \in I(E)$ such that $g=g_{1} g_{2}$. By Theorem 3.1,

$$
\Phi\left(L^{1}\left(\mathbb{R}^{+}\right)\right) A(\overline{\mathbb{D}})=I(E),
$$

so there exist $h \in L^{1}\left(\mathbb{R}^{+}\right)$and $f_{1} \in A(\overline{\mathbb{D}})$ such that $g_{1}=\Phi(h) f_{1}$. Hence $g=\Phi(h) f$ where $f=g_{2} f_{1} \in I(E)$. Then

$$
\breve{\Phi}\left(\mu_{\alpha}\right) g=\breve{\Phi}\left(\mu_{\alpha}\right) \Phi(h) f=\breve{\Phi}\left(\mu_{\alpha} * h\right) f=\int_{0}^{\infty} v^{t} f d\left(\mu_{\alpha} * h\right)(t) \text {. }
$$

Since $\mu_{\alpha} * h \rightarrow 0$ in $L^{1}\left(\mathbb{R}^{+}\right)$, it follows that $\breve{\Phi}\left(\mu_{\alpha}\right) g \rightarrow 0$ in $A(\overline{\mathbb{D}})$, so $\breve{\Phi}\left(\mu_{\alpha}\right) \rightarrow 0$ strongly in $A(\overline{\mathbb{D}} \backslash E)$.

Finally, since $L^{1}(\omega)$ is dense in $L^{1}\left(\mathbb{R}^{+}\right)$and $A(\overline{\mathbb{D}})$ is closed in $H^{\infty}(\mathbb{D})$ the last statement follows.

We will need the following three results on the weak-star topology on $H^{\infty}(\mathbb{D})$ and its connection to the strong topology on $A(\overline{\mathbb{D}} \backslash E)$ (regarded as the multiplier algebra of $I(E))$. 
LEMMA 3.3. If $\left(f_{\alpha}\right)$ is a net in $H^{\infty}(\mathbb{D})$ and $f_{\alpha} \rightarrow 0$ weak-star, then $f_{\alpha} \rightarrow 0$ pointwise on $\mathbb{D}$.

PROOF. For all $f \in H^{\infty}(\mathbb{D})$ and $z \in \mathbb{D}$,

$$
f(z)=\frac{1}{2 \pi} \int_{0}^{2 \pi} \frac{f\left(e^{i \theta}\right) e^{i \theta}}{e^{i \theta}-z} d \theta=\left\langle q_{z}, f\right\rangle,
$$

where $q_{z}(w)=w /(w-z)$ belongs to $L^{1}(\mathbb{T})$. The result follows.

LEMMA 3.4. Let $E \subseteq \mathbb{T}$ be a closed set of measure 0 , and $\left(f_{\alpha}\right)$ be a bounded net in $A(\overline{\mathbb{D}} \backslash E)$. Then $f_{\alpha} \rightarrow 0$ strongly if and only if $f_{\alpha} \rightarrow 0$ uniformly on compact subsets of $\mathbb{T} \backslash E$.

PROOF. First assume that $f_{\alpha} \rightarrow 0$ strongly. Given a compact subset $K \subset \mathbb{T} \backslash E$ and $\varepsilon>0$, choose $g \in I(E)$ with $|g-1|<\varepsilon$ on $K$. (For example, let $F$ be an outer function in $A(\overline{\mathbb{D}})$ such that $E=Z(F)$ and let $g=F^{t}$ for some $t>0$ sufficiently small.) Then choose $\alpha_{0}$ such that $\left\|f_{\alpha} g\right\|<\varepsilon$ for all $\alpha \geq \alpha_{0}$. It follows that, on $K$,

$$
\left|f_{\alpha}\right| \leq\left|f_{\alpha}(1-g)\right|+\left|f_{\alpha} g\right|<\left(\sup _{\alpha}\left\|f_{\alpha}\right\|+1\right) \varepsilon
$$

for all $\alpha \geq \alpha_{0}$, so $f_{\alpha} \rightarrow 0$ uniformly on $K$.

Assume conversely that $f_{\alpha} \rightarrow 0$ uniformly on compact subsets of $\mathbb{T} \backslash E$ and let $g \in I(E)$ and $\varepsilon>0$. Then

$$
K=\{z \in \mathbb{T}:|g(z)| \geq \varepsilon\}
$$

is a compact subset of $\mathbb{T} \backslash E$, so there exists $\alpha_{0}$ such that $\left|f_{\alpha}\right|<\varepsilon$ on $K$ for all $\alpha \geq \alpha_{0}$. We then have $\left|f_{\alpha} g\right|<\|g\| \varepsilon$ on $K$ and $\left|f_{\alpha} g\right|<\sup _{\alpha}\left\|f_{\alpha}\right\| \varepsilon$ on $\mathbb{T} \backslash K$ for all $\alpha \geq \alpha_{0}$, so $f_{\alpha} g \rightarrow 0$ in $A(\overline{\mathbb{D}})$. Hence $f_{\alpha} \rightarrow 0$ strongly.

COROLlary 3.5. Let $E \subseteq \mathbb{T}$ be a closed set of measure 0 . If a bounded net $\left(f_{\alpha}\right)$ in $A(\overline{\mathbb{D}} \backslash E)$ converges strongly to 0 , then it converges weak-star to 0 in $H^{\infty}(\mathbb{D})$.

PROOF. Let $q \in L^{1}(\mathbb{T})$ and $\varepsilon>0$. Choose an open neighbourhood $U$ of $E$ in $\mathbb{T}$ such that

$$
\int_{U}\left|q\left(e^{i \theta}\right)\right| d \theta<2 \pi \varepsilon
$$

By Lemma 3.4 we can choose $\alpha_{0}$ such that $\left|f_{\alpha}\right|<\varepsilon$ on $\mathbb{T} \backslash U$ for all $\alpha \geq \alpha_{0}$. Then

$$
\begin{aligned}
\left|\left\langle q, f_{\alpha}\right\rangle\right| & \leq \frac{1}{2 \pi} \int_{U}\left|q\left(e^{i \theta}\right) f_{\alpha}\left(e^{i \theta}\right)\right| d \theta+\frac{1}{2 \pi} \int_{\mathbb{T} \backslash U}\left|q\left(e^{i \theta}\right) f_{\alpha}\left(e^{i \theta}\right)\right| d \theta \\
& \left.<\sup _{\alpha}\left\|f_{\alpha}\right\|+\|q\|\right) \varepsilon
\end{aligned}
$$

for all $\alpha \geq \alpha_{0}$, so $f_{\alpha} \rightarrow 0$ weak-star in $H^{\infty}(\mathbb{D})$. 
We are now in a position to prove that the extension $\widetilde{\Phi}: M\left(\mathbb{R}^{+}\right) \rightarrow H^{\infty}(\mathbb{D})$ of a homomorphism $\Phi: L^{1}\left(\mathbb{R}^{+}\right) \rightarrow A(\overline{\mathbb{D}})$ is weak-star continuous. However, the case of a constant function $v \in A(\overline{\mathbb{D}} \backslash E)$ has to be excluded. If, for instance, $v \equiv 1$, then $\widetilde{\Phi}(\mu)=\mu\left(\mathbb{R}^{+}\right)$, so $\delta_{t} \rightarrow 0$ weak-star in $M\left(\mathbb{R}^{+}\right)$as $t \rightarrow \infty$, but $\widetilde{\Phi}\left(\delta_{t}\right)=1$ for all $t \in \mathbb{R}^{+}$, so $\widetilde{\Phi}$ is not weak-star continuous.

THEOREM 3.6. Let $\Phi: L^{1}\left(\mathbb{R}^{+}\right) \rightarrow A(\overline{\mathbb{D}})$ be a nonzero homomorphism and let

$$
\widetilde{\Phi}(\mu)=\int_{\mathbb{R}^{+}} v^{t} d \mu(t)
$$

for all $\mu \in M\left(\mathbb{R}^{+}\right)$be the unique extension to a homomorphism from $M\left(\mathbb{R}^{+}\right)$to $H^{\infty}(\mathbb{D})$ given by Theorem 3.1. Assume that the function $v \in A(\overline{\mathbb{D}} \backslash E)$ is not constant. Then:

(a) the homomorphism $\widetilde{\Phi}$ is weak-star continuous;

(b) the semigroup $\left(v^{t}\right)_{t \in \mathbb{R}^{+}}$is weak-star continuous in $H^{\infty}(\mathbb{D})$ and $v^{t} \rightarrow 0$ weakstar as $t \rightarrow \infty$;

(c) for all $q \in L^{1}(\mathbb{T})$ and $t \in \mathbb{R}^{+}$let

$$
(T q)(t)=\left\langle q, v^{t}\right\rangle=\frac{1}{2 \pi} \int_{0}^{2 \pi} q\left(e^{i \theta}\right) v^{t}\left(e^{i \theta}\right) d \theta
$$

which defines a bounded linear operator

$$
T: L^{1}(\mathbb{T}) / H_{0}^{1}(\mathbb{D}) \rightarrow C_{0}\left(\mathbb{R}^{+}\right)
$$

with $T^{*}=\widetilde{\Phi}$.

Proof. For all $q \in L^{1}(\mathbb{T})$ and $\mu \in M\left(\mathbb{R}^{+}\right)$,

$$
\int_{\mathbb{R}^{+}}\left|\left\langle q, v^{t}\right\rangle\right| d \mu(t) \leq \frac{1}{2 \pi} \int_{\mathbb{R}^{+}} \int_{\mathbb{T}}\left|q\left(e^{i \theta}\right)\right|\left|\nu^{t}\left(e^{i \theta}\right)\right| d \theta d \mu(t)<\infty,
$$

since $|\nu| \leq 1$. Hence $\int_{\mathbb{R}^{+}}\left\langle q, v^{t}\right\rangle d \mu(t)$ exists, and by Fubini's theorem,

$$
\begin{aligned}
\int_{\mathbb{R}^{+}}\left\langle q, v^{t}\right\rangle d \mu(t) & =\frac{1}{2 \pi} \int_{\mathbb{R}^{+}} \int_{\mathbb{T}} q\left(e^{i \theta}\right) v^{t}\left(e^{i \theta}\right) d \theta d \mu(t) \\
& =\frac{1}{2 \pi} \int_{\mathbb{T}_{\mathbb{R}^{+}}} \int_{\left.\mathbb{R}^{i \theta}\right) v^{t}\left(e^{i \theta}\right) d \mu(t) d \theta} \\
& =\left\langle q, \int_{\mathbb{R}^{+}} v^{t} d \mu(t)\right\rangle .
\end{aligned}
$$

We can therefore apply Proposition 2.2 and thus only need to prove part (b).

By Theorem 3.1 the semigroup $\left(v^{t}\right)_{t \in \mathbb{R}^{+}}$is bounded and strongly continuous in $A(\overline{\mathbb{D}} \backslash E)$, so it follows from Corollary 3.5 that it is weak-star continuous in $H^{\infty}(\mathbb{D})$. Moreover, since $|v| \leq 1$ on $\overline{\mathbb{D}}$ and $v$ is not constant, $|v|<1$ in $\mathbb{D}$, and thus $v^{t} \rightarrow 0$ pointwise on $\mathbb{D}$ as $t \rightarrow \infty$. By Lemma 3.3 we therefore conclude that 0 is the only weak-star cluster point of the bounded net $\left(v^{t}\right)$ as $t \rightarrow \infty$, so $v^{t} \rightarrow 0$ weak-star as $t \rightarrow \infty$. 
We note that the fact that $v^{t} \rightarrow 0$ weak-star as $t \rightarrow \infty$ in the previous theorem cannot be deduced from Corollary 3.5, since we do not necessarily have $v^{t} \rightarrow 0$ strongly as $t \rightarrow \infty$. For example, if

$$
v^{t}(z)=\exp \left(-t \frac{1+z}{1-z}\right),
$$

then the semigroup $\left(v^{t}\right)_{t \in \mathbb{R}^{+}}$, arising from the Laplace transform (mentioned after Theorem 3.1), acts by isometries on $I(\{1\})$ and therefore $v^{t}$ does not tend to 0 strongly as $t \rightarrow \infty$.

COROllary 3.7. Let $\omega$ be an algebra weight, let $\Phi: L^{1}(\omega) \rightarrow A(\overline{\mathbb{D}})$ be a nonzero homomorphism and let

$$
\widetilde{\Phi}(\mu)=\int_{\mathbb{R}^{+}} v^{t} d \mu(t)
$$

for all $\mu \in M(\omega)$ be the unique extension to a homomorphism from $M(\omega)$ to $H^{\infty}(\mathbb{D})$ given by Theorem 3.1. Assume that the function $v \in A(\overline{\mathbb{D}} \backslash E)$ is not constant. Then the following hold.

(a) The homomorphism $\widetilde{\Phi}$ is weak-star continuous.

(b) The semigroup $\left(v^{t}\right)_{t \in \mathbb{R}^{+}}$is weak-star continuous in $H^{\infty}(\mathbb{D})$ and $v^{t} / \omega(t) \rightarrow 0$ weak-star as $t \rightarrow \infty$.

(c) For all $q \in L^{1}(\mathbb{T})$ and $t \in \mathbb{R}^{+}$let

$$
(T q)(t)=\left\langle q, v^{t}\right\rangle=\frac{1}{2 \pi} \int_{0}^{2 \pi} q\left(e^{i \theta}\right) v^{t}\left(e^{i \theta}\right) d \theta .
$$

This defines a bounded linear operator $T: L^{1}(\mathbb{T}) / H_{0}^{1}(\mathbb{D}) \rightarrow C_{0}(1 / \omega)$ with $T^{*}=\widetilde{\Phi}$.

Proof. As in the proof of Theorem 3.6 we can apply Proposition 2.2 and thus only need to prove part (a).

We may assume that $L^{1}(\omega)$ is semisimple (see the end of Section 2) and hence that

$$
r=\lim _{t \rightarrow \infty} \omega(t)^{1 / t}>0 .
$$

Setting $\widetilde{\omega}(t)=r^{-t} \omega(t)$ for all $t \in \mathbb{R}^{+}$, we have $\lim _{t \rightarrow \infty} \widetilde{\omega}(t)^{1 / t}=1$ and the map $f(t) \mapsto f(t) r^{t}$ is a norm and weak-star continuous isomorphism between $L^{1}(\omega)$ and $L^{1}(\widetilde{\omega})$. Without loss of generality we may therefore assume that $r=1$. Hence $\Phi$ extends to $\breve{\Phi}: M\left(\mathbb{R}^{+}\right) \rightarrow H^{\infty}(\mathbb{D})$ by Proposition 3.2, and $\breve{\Phi}$ is weak-star continuous by Theorem 3.6. The extension $\widetilde{\Phi}: M(\omega) \rightarrow H^{\infty}(\mathbb{D})$ given by Theorem 3.1 is the restriction of $\breve{\Phi}$ to $M(\omega)$ and is therefore weak-star continuous.

\section{Homomorphisms into the algebra of absolutely convergent Taylor series and into Beurling algebras}

In this section we turn our attention to homomorphisms from $L^{1}(\omega)$ into the Wiener algebra of absolutely convergent Taylor series on $\overline{\mathbb{D}}$ or into weighted analogues. 
For these algebras the extension provided by Proposition 2.1 actually maps into the algebra itself. Moreover, the algebras are dual spaces and we will show that the extension is weak-star continuous.

We denote by $A^{+}$the Wiener algebra of those functions $f$ on $\overline{\mathbb{D}}$ with absolutely convergent Taylor series with the norm

$$
\|f\|=\sum_{n=0}^{\infty}|\widehat{f}(n)|,
$$

where $\widehat{f}(n)$ for all $n \in \mathbb{N}_{0}$ are the Taylor coefficients of $f$.

The Beurling algebras are defined similarly. Let $v=\left(v_{n}\right)_{n \in \mathbb{N}_{0}}$ be an algebra weight on $\mathbb{N}_{0}$, that is, a positive sequence such that $v_{0}=1$ and $v_{n+m} \leq v_{n} v_{m}$ for all $n, m \in \mathbb{N}_{0}$. In this paper, we will consider only semisimple Beurling algebras with character space $\overline{\mathbb{D}}$, so we further assume that $\lim _{n \rightarrow \infty} \underline{v}_{n}^{1 / n}=1$. We then define the Beurling algebra $A_{v}^{+}$to consist of those functions $f$ on $\overline{\mathbb{D}}$ for which

$$
\|f\|=\sum_{n=0}^{\infty}|\widehat{f}(n)| v_{n}<\infty .
$$

It is well known that $A^{+}$and $A_{v}^{+}$are Banach algebras with character space $\overline{\mathbb{D}}$.

We can identify $A_{v}^{+}$with the dual space of $c_{0}(1 / v)$ (the Banach space of sequences $\left(x_{n}\right)_{n \in \mathbb{N}_{0}}$ with $x_{n} / v_{n} \rightarrow 0$ as $n \rightarrow \infty$ ), with the duality defined by

$$
\left\langle\left(x_{n}\right), f\right\rangle=\sum_{n=0}^{\infty} x_{n} \widehat{f}(n)
$$

for all $\left(x_{n}\right) \in c_{0}(1 / v)$ and $f \in A_{v}^{+}$. When $v \equiv 1$, this identifies $A^{+}$with the dual space of $c_{0}$.

For a homomorphism $\Phi: L^{1}\left(\mathbb{R}^{+}\right) \rightarrow A^{+}$, we proved in [8, Theorems 3.1 and 3.3] that the semigroup $\left(v^{t}\right)_{t \in \mathbb{R}^{+}}$of Proposition 2.1 actually belongs to $A^{+}$and has finite zero set. The argument turns out to hold for homomorphisms $\Phi: L^{1}(\omega) \rightarrow A^{+}$as well. In order to present a slightly extended result in Theorem 4.2 below, we need the following lemma.

LEMMA 4.1. Let $E \subseteq \mathbb{T}$ be a finite set. Then $\iota$, given by

$$
\iota(f) g=f g
$$

for all $f \in A^{+}$and $g \in I(E)$, is an isomorphism between $A^{+}$and $\operatorname{Mul}(I(E))$.

PROOF. It is clear that $\iota$ is an injective homomorphism from $A^{+}$to $\operatorname{Mul}(I(E))$. Since, for instance, $\left((1-z)^{1 / n}\right)_{n \in \mathbb{N}}$ is a bounded approximate identity for $I(\{1\})$, it follows that $I(E)$ has a bounded approximate identity $\left(e_{n}\right)$. Let $T \in \operatorname{Mul}(I(E))$ and $g \in I(E)$. The bounded sequence $T\left(e_{n}\right)$ in $A^{+}$has a weak-star convergent subsequence $T\left(e_{n_{k}}\right)$ with limit $f \in A^{+}$as $k \rightarrow \infty$. It is well known (see, for instance, [9, Corollary 3.2]) that multiplication in $A^{+}$is separately weak-star continuous. Since $T$ is a multiplier it 
follows that

$$
T\left(e_{n_{k}} g\right)=T\left(e_{n_{k}}\right) g \rightarrow f g
$$

weak-star in $A^{+}$as $k \rightarrow \infty$. On the other hand, $T\left(e_{n_{k}} g\right) \rightarrow T(g)$ in $A^{+}$(in norm) as $k \rightarrow \infty$, so we deduce that $T(g)=f g$, which finishes the proof.

We remark in passing that the strong topology on $A^{+} \simeq \operatorname{Mul}(I(E))$ depends on the set $E$. For instance, $(1-z)^{1 / n} \rightarrow 1$ strongly in $\operatorname{Mul}(I(\{1\}))$ as $n \rightarrow \infty$, but not in $\operatorname{Mul}\left(A^{+}\right)$(which corresponds to $E=\emptyset$ ).

THEOREM 4.2. Let $\omega$ be an algebra weight, let $\Phi: L^{1}(\omega) \rightarrow A^{+}$be a nonzero homomorphism and let $\mathcal{C}$ be the closed ideal $\Phi\left(L^{1}(\omega)\right) A^{+}$. Then there exists a finite set $E \subset \mathbb{T}$ such that

$$
\mathcal{C}=I(E)=\left\{g \in A^{+}: g=0 \text { on } E\right\},
$$

and there exists a semigroup $\left(v^{t}\right)_{t \in \mathbb{R}^{+}}$in $\mathcal{C}$ which is continuous in $(0, \infty)$ and strongly continuous at 0 (considered as a semigroup in $\operatorname{Mul}(\mathcal{C})$ ) such that

$$
\widetilde{\Phi}(\mu)=\int_{\mathbb{R}^{+}} v^{t} d \mu(t)
$$

exists as a Bochner integral in $A^{+}$for all $\mu \in M(\omega)$ and $\widetilde{\Phi}: M(\omega) \rightarrow A^{+}$is a homomorphism that uniquely extends $\Phi$ and is norm as well as strongly continuous (as a map into $\operatorname{Mul}(\mathcal{C})$ ).

PROOF. Most of the proof is identical to that of [8, Theorems 3.1 and 3.3]. The statements about strong continuity follow from the previous lemma and Proposition 2.1.

It is not hard to see that the semigroup $\left(v^{t}\right)_{t \in \mathbb{R}^{+}}$in the previous theorem is not continuous at 0 if $E \neq \emptyset$ (for instance, if $\left.v^{t}(z)=((1-z) / 2)^{t}\right)$.

For homomorphisms into $A_{v}^{+}$we can say even more than in Theorem 4.2. We first need the fact that weak-star convergence in $A_{v}^{+}$implies pointwise convergence.

LEMMA 4.3. Suppose that $\left(f_{\alpha}\right)$ be a net in $A_{v}^{+}$and that $f_{\alpha} \rightarrow 0$ weak-star in $A_{v}^{+}$. Then $f_{\alpha} \rightarrow 0$ pointwise on $\mathbb{D}$. Further, if $v_{n} \rightarrow \infty$ as $n \rightarrow \infty$, then $f_{\alpha} \rightarrow 0$ pointwise on $\overline{\mathbb{D}}$.

PROOF. We have $\left(z^{n}\right)_{n \in \mathbb{N}_{0}} \in c_{0}(1 / v)$ for all $z \in \mathbb{D}$. Also $\left(z^{n}\right)_{n \in \mathbb{N}_{0}} \in c_{0}(1 / v)$ for all $z \in \overline{\mathbb{D}}$ if $v_{n} \rightarrow \infty$ as $n \rightarrow \infty$. Thus

$$
\left\langle\left(z^{n}\right)_{n \in \mathbb{N}_{0}}, f\right\rangle=\sum_{0}^{\infty} \widehat{f}(n) z^{n}=f(z)
$$

for all $f \in A_{v}^{+}$, and the result follows. 
THEOREM 4.4. Let $\omega$ be an algebra weight on $\mathbb{R}^{+}$, let $v$ be an algebra weight on $\mathbb{N}_{0}$ and assume that $v_{n} \rightarrow \infty$ as $n \rightarrow \infty$. Let $\Phi: L^{1}(\omega) \rightarrow A_{v}^{+}$be a nonzero homomorphism. Then $\Phi\left(L^{1}(\omega)\right)=A_{v}^{+}$, and there exists a continuous semigroup $\left(v^{t}\right)_{t \in \mathbb{R}^{+}}$in $A_{v}^{+}$such that

$$
\widetilde{\Phi}(\mu)=\int_{\mathbb{R}^{+}} v^{t} d \mu(t)
$$

exists as a Bochner integral in $A_{v}^{+}$for all $\mu \in M(\omega)$ and $\widetilde{\Phi}: M(\omega) \rightarrow A_{v}^{+}$is a homomorphism that uniquely extends $\Phi$ and is continuous when $M(\omega)$ is equipped with the strong topology and $A_{v}^{+}$with the norm topology. Moreover, the zero set of $v$ is empty, so $v^{t}$ is invertible for all $t \in \mathbb{R}^{+}$and the semigroup extends to a continuous group $\left(v^{t}\right)_{t \in \mathbb{R}}$ in $A_{v}^{+}$.

PROOF. Since $L^{1}(\omega)$ has a bounded approximate identity, it follows from a result of Sinclair (see [11] or [1, Corollary 2.9.43]) that it contains a bounded semigroup $\left(\eta^{s}\right)_{s>0}$ such that $\left(\eta^{1 / n}\right)_{n \in \mathbb{N}}$ is a sequential bounded approximate identity for $L^{1}(\omega)$. In particular, $\Phi(\eta) \neq 0$. Let $E \subset \mathbb{T}$ be the zero set of $\Phi(\eta)$. Then $\Phi(\eta)^{1 / n} \rightarrow 1_{\mathbb{T} \backslash E}$ pointwise on $\overline{\mathbb{D}}$ as $n \rightarrow \infty$. The bounded sequence $\left(\Phi(\eta)^{1 / n}\right)_{n \in \mathbb{N}}$ has a weak-star clusterpoint $f \in A_{v}^{+}$as $n \rightarrow \infty$, and it follows from Lemma 4.3 that $f=1_{\mathbb{T} \backslash E}$. Hence $\Phi(\eta)$ has no zeros on $\overline{\mathbb{D}}$ and is therefore invertible in $A_{v}^{+}$, so $\Phi\left(L^{1}(\omega)\right)=A_{v}^{+}$. In particular, $\mathcal{C}=\Phi\left(L^{1}(\omega)\right) A_{v}^{+}=A_{v}^{+}$and thus $\operatorname{Mul}(\mathcal{C})=A_{v}^{+}$. By Proposition 2.1 the semigroup $\left(v^{t}\right)_{t \in \mathbb{R}^{+}}$is therefore continuous in $A_{v}^{+}$with $\left\|v^{t}\right\|=O(\omega(t))$ as $t \rightarrow \infty$. Hence

$$
\widetilde{\Phi}(\mu)=\int_{\mathbb{R}^{+}} v^{t} d \mu(t)
$$

for all $\mu \in M(\omega)$ defines a homomorphism $\widetilde{\Phi}: M(\omega) \rightarrow A_{v}^{+}$with the properties required. (The fact that $v^{t} \in A_{v}^{+}$can also be seen by showing that it is a weak-star clusterpoint of the bounded sequence $\Phi\left(\delta_{t} * \eta^{1 / n}\right)$ as $n \rightarrow \infty$.)

For the extensions from Theorems 4.2 and 4.4 the obvious dual space $Y^{*}$ in Proposition 2.2 is the algebra, $A^{+}$or $A_{v}^{+}$, itself. We will now prove that these extensions are weak-star continuous. We will need the following result.

LEMMA 4.5. Let $\left(f_{\alpha}\right)$ be a bounded net in $A_{v}^{+}$with $\lim _{\alpha} \widehat{f}_{\alpha}(n)=0$ for all $n \in \mathbb{N}_{0}$. Then $\lim _{\alpha} f_{\alpha}=0$ weak-star in $A_{v}^{+}$.

Proof. Let $x \in c_{0}(1 / v)$. Given $\varepsilon>0$, choose $N \in \mathbb{N}$ such that $\left|x_{n}\right| / v_{n}<\varepsilon$ for all $n>N$, and then choose $\alpha_{0}$ such that

$$
\sum_{n=0}^{N}\left|\widehat{f}_{\alpha}(n)\right| v_{n}<\varepsilon
$$

for all $\alpha \geq \alpha_{0}$. Then

$$
\left|\left\langle x, f_{\alpha}\right\rangle\right| \leq \sum_{n=0}^{N} \frac{\left|x_{n}\right|}{v_{n}} \cdot\left|\widehat{f}_{\alpha}(n)\right| v_{n}+\sum_{n=N+1}^{\infty} \frac{\left|x_{n}\right|}{v_{n}} \cdot\left|\widehat{f}_{\alpha}(n)\right| v_{n}<\left(\|x\|+\sup _{\alpha}\left\|f_{\alpha}\right\|\right) \varepsilon
$$

for all $\alpha \geq \alpha_{0}$, and the result follows. 
In the following theorem the case of a constant function $v$ has to be excluded using the same argument as in Theorem 3.6. Also note that bounded weights $v$ correspond to the algebra $A^{+}$.

THEOREM 4.6. Let $\omega$ be an algebra weight on $\mathbb{R}^{+}$and let $v$ be an algebra weight on $\mathbb{N}_{0}$ which is either bounded or has $v_{n} \rightarrow \infty$ as $n \rightarrow \infty$. Let $\Phi: L^{1}(\omega) \rightarrow A_{v}^{+}$be $a$ nonzero homomorphism and let $\widetilde{\Phi}$, given by

$$
\widetilde{\Phi}(\mu)=\int_{\mathbb{R}^{+}} v^{t} d \mu(t)
$$

for all $\mu \in M(\omega)$, be the unique extension to a homomorphism from $M(\omega)$ to $A_{v}^{+}$given by Theorems 4.2 or 4.4. Assume that the function $v \in A_{v}^{+}$is not constant. Then:

(a) the homomorphism $\widetilde{\Phi}$ is weak-star continuous;

(b) the semigroup $\left(v^{t}\right)_{t \in \mathbb{R}^{+}}$is weak-star continuous in $A_{v}^{+}$and $v^{t} / \omega(t) \rightarrow 0$ weakstar in $A_{v}^{+}$as $t \rightarrow \infty$;

(c) for all $x \in c_{0}(1 / v)$ and $t \in \mathbb{R}^{+}$let

$$
(T x)(t)=\left\langle x, v^{t}\right\rangle=\sum_{n=0}^{\infty} x_{n} \widehat{v^{t}}(n),
$$

which defines a bounded linear operator $T: c_{0}(1 / v) \rightarrow C_{0}(1 / \omega)$ with $T^{*}=\widetilde{\Phi}$. ProOF. For all $\mu \in M(\omega)$ we have $\widetilde{\Phi}(\mu)=\int_{\mathbb{R}^{+}} v^{t} d \mu(t)$ as a Bochner integral and, in particular, as a weak-star Bochner integral. Moreover,

$$
\langle y, \widetilde{\Phi}(\mu)\rangle=\int_{\mathbb{R}^{+}}\left\langle y, v^{t}\right\rangle d \mu(t)
$$

for all $y \in c_{0}(1 / v)$ since Bochner integrals commute with continuous linear functionals [6, Theorem 3.7.12]. Hence the condition in Proposition 2.2 is satisfied, so we only need to prove part (b).

If $v_{n} \rightarrow \infty$ as $n \rightarrow \infty$, then $v^{t}$ is continuous and, in particular, weak-star continuous in $A_{v}^{+}$for $t \in \mathbb{R}^{+}$by Theorem 4.4. If $v$ is bounded, then we may assume that $v \equiv 1$. Then $v^{t}$ is continuous and, in particular, weak-star continuous in $A^{+}$for all $t>0$ by Theorem 4.4, so we only need to prove weak-star continuity at $t=0$. We have $v^{t}(z)=e^{-t G(z)} \rightarrow 1$ uniformly on compact subsets of $\mathbb{T} \backslash E$. Now $v^{t}$ is uniformly bounded on $\mathbb{T}$, so $v^{t} \rightarrow 1$ in $L^{1}(\mathbb{T})$ as $t \rightarrow 0$. Hence $\widehat{v^{t}}(n) \rightarrow \widehat{1}(n)$ as $t \rightarrow 0$ for all $n \in \mathbb{N}_{0}$, so it follows from Lemma 4.5 that $v^{t} \rightarrow 1$ weak-star in $A^{+}$as $t \rightarrow 0$, that is, $v^{t}$ is weak-star continuous in $A_{v}^{+}$at $t=0$.

In order to prove that $v^{t} / \omega(t) \rightarrow 0$ weak-star in $A_{v}^{+}$as $t \rightarrow \infty$ we first observe that

$$
C=\sup _{|z| \leq 1 / 2}|v(z)|<1
$$

since $v$ is not constant. By Cauchy's estimates (see, for instance, [10, Theorem 10.26]),

$$
\left|\widehat{v^{t}}(n)\right|=\frac{\left|\left(v^{t}\right)^{(n)}(0)\right|}{n !} \leq \frac{C^{t}}{\left(\frac{1}{2}\right)^{n}} \rightarrow 0 \quad \text { as } t \rightarrow \infty
$$


for all $n \in \mathbb{N}_{0}$. Since $v^{t} / \omega(t)$ is bounded in $A_{v}^{+}$, it follows from Lemma 4.5 that $v^{t} / \omega(t) \rightarrow 0$ weak-star in $A_{v}^{+}$as $t \rightarrow \infty$.

\section{References}

[1] H. G. Dales, Banach Algebras and Automatic Continuity, London Mathematical Society Monographs, New Series, 24 (Oxford University Press, Oxford, 2000).

[2] J. Esterle, 'Quasimultipliers, representations of $H^{\infty}$, and the closed ideal problem for commutative Banach algebras', in: Radical Banach Algebras and Automatic Continuity, Lecture Notes in Mathematics, 975 (eds. J. M. Bachar et al.) (Springer, Berlin, 1983), pp. 66-162.

[3] F. Ghahramani, J. P. McClure and S. Grabiner, 'Standard homomorphisms and regulated weights on weighted convolution algebras', J. Funct. Anal. 91 (1990), 278-286.

[4] S. Grabiner, 'Homomorphisms and semigroups in weighted convolution algebras', Indiana Univ. Math. J. 37 (1988), 589-615.

[5] S. Grabiner, 'Weak * properties of weighted convolution algebras II', Preprint, 2006.

[6] E. Hille and R. S. Phillips, Functional Analysis and Semi-Groups, American Mathematical Society Colloquium Publications, 31 (American Mathematical Society, Providence, RI, 1957), revised edition.

[7] K. Hoffman, Banach Spaces of Analytic Functions (Prentice Hall, Englewood Cliffs, NJ, 1962).

[8] T. V. Pedersen, 'Sinclair homomorphisms and semigroups of analytic functions', J. Funct. Anal. 145 (1997), 527-554.

[9] T. V. Pedersen, 'Banach algebras weak* ${ }^{*}$ generated by their idempotents', Proc. Edinb. Math. Soc. 45 (2002), 681-692.

[10] W. Rudin, Real and Complex Analysis, 3rd edn (McGraw-Hill, New York, 1987).

[11] A. M. Sinclair, Continuous Semigroups in Banach Algebras, London Mathematical Society Lecture Note Series, 63 (Cambridge University Press, Cambridge, 1982).

\footnotetext{
THOMAS VILS PEDERSEN, Department of Natural Sciences and Environment, Faculty of Life Sciences, University of Copenhagen, Thorvaldsensvej 40, DK-1871 Frederiksberg C, Denmark

e-mail: vils@life.ku.dk
} 